\title{
Charge-coupled device spectra of the Galilean satellites: Molecular oxygen on Ganymede
}

\author{
John R. Spencer \\ Lowell Observatory, Flagstaff, Arizona \\ Wendy M. Calvin \\ U.S. Geological Survey, Flagstaff, Arizona \\ Michael J. Person \\ Lowell Observatory, Flagstaff, Arizona
}

\begin{abstract}
We have obtained 3200-7800 $\AA$ CCD spectra of the Galilean satellites at a variety of orbital longitudes, with spectral resolution between 3 and $18 \AA$ and signal-tonoise ratios of up to 2000 at $6000 \AA$. Despite the higher resolution and signal-to-noise ratio than previous published spectra, no new features are seen on Io, Europa, and Callisto. However, Ganymede shows an unusual and previously unreported shallow absorption feature at $5773 \AA$, which is much stronger on the trailing side, and a weaker band at 6275 $\AA$. The features are apparently due to diatomic oxygen, and require simultaneous electronic transitions in two adjacent molecules: they are the strongest visible-wavelength absorption bands in solid or liquid oxygen. Because condensed pure oxygen is not stable at Ganymede surface temperatures and pressures, the oxygen must be trapped in other surface materials, perhaps in water ice, with the constraint that $\mathrm{O}_{2}$ molecules must be close enough together for simultaneous electronic transitions. Magnetospheric bombardment of water ice is a plausible production mechanism for the $\mathrm{O}_{2}$. Continuum shapes on all four satellites are more clearly seen than in previous spectra. The shape of the continuum is very similar on Ganymede and Callisto but is strikingly different on Europa, indicating a different origin for the nonice component on Europa. The shape of the Europa continuum suggests that allotropes or compounds of sulfur may be the dominant spectrally active materials in the visible spectrum. No convincing changes since 1978 are visible in Io's spectrum, despite the high resurfacing rates, suggesting that volcanic resurfacing tends to overpaint areas with more material of the same composition.
\end{abstract}

\section{Motivation}

No published visible-wavelength spectra of the Galilean satellites have been taken since 1978 , before the advent of modern charge-coupled device (CCD) spectrographs. The best published spectra are from Nelson and Hapke [1978], with a spectral resolution of $40 \AA$ and signal-to-noise ratio $(\mathrm{S} / \mathrm{N})$ of about 100 . Other early spectra [Johnson and McCord, 1970; Johnson, 1971; McFadden et al., 1980] have lower resolution, though the McFadden et al. spectra have excellent orbital longitude coverage. We have therefore obtained new CCD spectra of the satellites in order to (1) provide better spectra of Io, to help distinguish between competing models for its surface composition [Hapke 1989; Moses and Nash 1991; Nash 1993]; (2) look for changes in Io's spectrum since the late 1970s; (3) improve our understanding of the nonice components on the icy satellite surfaces, which are best seen at visible and UV wavelengths; and (4) look for new spectral features.

\section{Copyright 1995 by the American Geophysical Union.}

Paper number 95JE01503.

0148-0227/95/95JE-01503\$05.00

\section{Observations}

On March 19-20 (UT), 1993, we used the Ohio State University (OSU) Boller and Chivens CCD long-slit grating spectrograph at the Perkins 72-inch telescope at Lowell Observatory, with a low-resolution grating (150 lines $\mathrm{mm}^{-1}$ ) that allowed coverage of the 3250 to $7800-\AA$ region with two grating positions. A slit width of 5 arc sec was normally used, providing a dispersion of $6 \AA$ per pixel and an effective spectral resolution of about $18 \AA$. All of the Galilean satellites were observed, as well as the solar-type standard stars 16 Cyg B, 35 Leo, and HR 3538 [Hardorp 1980]. We typically took batches of 10 or 20 consecutive spectra of the same object with the same grating position, each with an exposure of 1 or $2 \mathrm{~s}$, in order to build up $S / N$. The air mass range for the Galilean satellites was $1.27-1.40$, bracketed by the standards. Wavelength calibration was provided by an internal FeNe lamp, and checked against Fraunhofer lines in the satellite and comparison star spectra.

Following the discovery of the $5773-\AA$ band on Ganymede (see below), we borrowed telescope time to make follow-up observations of possible discrete features in the spectra of the Galilean satellites in twilight in April and July 1993. Time did not permit standard star observations on these 
runs: instead the satellites were ratioed to each other to remove the Fraunhofer lines and isolate features intrinsic to the satellites. Because we were only interested in discrete spectral features and not continuum slopes, the lack of good air mass corrections and standard stars on these twilight runs was only a problem at the wavelengths of discrete telluric absorption bands: there are no significant telluric bands near the $5770-\AA$ region that we were most interested in. For some of these twilight observations, we used a 350 line $\mathrm{mm}^{-1}$ grating, with a 2 arc sec slit, giving $2.6 \AA$ pixel $^{-1}$ and a resolution of about $6 \AA$ over the 5400 - to $6600-\AA$ range.

In March and April 1994 we obtained many additional spectra which extended the longitude coverage on Ganymede and the other satellites. A slit width of 3 arc sec or narrower was used for maximum spectral resolution. On April 5, 1994 we used a grating with the relatively high dispersion of $1 \AA$ pixel $^{-1}$, with a 1 arc sec slit, for a close look at the Ganymede 5770- and 6250- $\AA$ features. See Table 1 for details.

\section{Reduction}

Individual spectra were extracted from the CCD frames in the following manner. First, a bias frame was subtracted from each CCD frame and the frame was divided by a flat field, obtained by illuminating the array with a continuum lamp internal to the spectrograph and using the same grating position as the object spectrum. Bad pixels were removed using a routine that automatically replaced pixels that were different from their surroundings by a preset threshold with a median of their immediate neighbors. An "object strip" was defined that normally extended 10 pixels to either side of the center line of the object spectrum, wide enough to include all significant light from the object itself, and "sky strips" were defined that extended from the margins of the object strip a further 20 or 30 pixels from the object centerline. At each row of the image perpendicular to the dispersion direction, a third-order polynomial (fourthorder for most of the 1994 data) was simultaneously fit to the sky strip values on both sides of the object strip. The value of this polynomial was evaluated at each pixel of the object strip and was subtracted from that pixel. Finally, the values of all sky-subtracted pixels in the object strip were summed to give the value of the extracted spectrum at that row of the image.

Each extracted spectrum from each batch of consecutive images of a single object was automatically registered in wavelength to a reference spectrum from the same batch, to a precision of about 0.2 pixels, by ratioing to the reference spectrum and shifting (using sinc interpolation) to find the best cancellation of the Fraunhofer lines in the ratio. Because, especially with the narrower slit widths, some spectra had anomalous continuum slopes due the effect of guiding errors on a object that was smeared out on the sky by differential refraction, spectra whose slope differed markedly from others in the same batch were rejected. The remainder were averaged together to produce a single high $\mathrm{S} / \mathrm{N}$ spectrum. Final errors in spectrum slope due to differential refraction are probably $<10 \%$ of the continuum level over the whole wavelength range.

Due to limitations of the data, many of which were taken in nonphotometric conditions, atmospheric extinction was handled only approximately. Extinction was determined by first ratioing standard star, or Callisto, spectra taken at different air masses: Callisto rotates only $4^{\circ}$ in 7 hours, so it could be safely assumed to be intrinsically constant over most of a night. Shortward of $6000 \AA$, where extinction varies smoothly with wavelength, we fit the ratio spectrum with a fourth-order polynomial to determine the extinction coefficient at each wavelength, assuming zero extinction at $6000 \AA$. Longward of $6000 \AA$, where extinction is dominated by discrete bands, we used the ratio of the two standard star spectra to determine the extinction coefficient directly within the wavelength bands $6491-6615 \AA, 6800$ $7030 \AA, 7108-7347 \AA$, and $7515-7720 \AA$, where the strongest telluric features are located, and assumed zero extinction outside these bands. On the dates of the spectra for which we present continuum slopes, March 19 and 20, 1993, the air mass difference between the Galilean satellites and the comparison stars was less than 0.35 air masses, so the crudeness of the extinction correction has a relatively small effect on the quality of the reduction. The success of the extinction

Table 1. Log of Observations

\begin{tabular}{|c|c|c|c|c|c|c|c|c|}
\hline \multirow[b]{2}{*}{ Date } & \multicolumn{2}{|c|}{ Orbital } & \multicolumn{2}{|c|}{ Phase } & \multirow{2}{*}{$\begin{array}{c}\text { Solar } \\
\text { Phase } \\
\text { Angle, deg }\end{array}$} & \multicolumn{3}{|c|}{ Grating } \\
\hline & Io & $\begin{array}{c}\text { Eur- } \\
\text { opa }\end{array}$ & $\begin{array}{l}\text { Gany- } \\
\text { mede }\end{array}$ & $\begin{array}{c}\text { Cal- } \\
\text { listo }\end{array}$ & & $\begin{array}{l}\text { Standard } \\
\text { Stars }\end{array}$ & $\begin{array}{c}\text { Dispersion, } \\
\AA_{\text {pixel }^{-1}}\end{array}$ & $\begin{array}{l}\text { Air Mass } \\
\text { Range }\end{array}$ \\
\hline March 19, 1993 & $52-70$ & - & - & - & 2.3 & $\begin{array}{c}\text { 35 Leo, } \\
\text { HR } 3538\end{array}$ & 6.0 & $1.21-1.34$ \\
\hline March 20, 1993 & $253-276$ & $78-79$ & $252-253$ & 70 & 2.0 & $\begin{array}{c}35 \text { Leo, } \\
16 \text { Cyg B, } \\
\text { HR } 3538\end{array}$ & 6.0 & $1.27-1.66$ \\
\hline April 22, 1993 & - & - & $104-105$ & 62 & 4.5 & - & 2.6 & $1.85-2.24$ \\
\hline July 19,1993 & - & - & 215 & 160 & 10.2 & - & 2.6 & $2.11-2.50$ \\
\hline July 28, 1993 & - & 275 & 306 & - & 9.6 & - & 6.0 & $2.07-2.34$ \\
\hline March 29, 1994 & - & - & 328 & 189 & -6.0 & - & 6.0 & $1.98-2.11$ \\
\hline March 30,1994 & - & - & $11-17$ & $210-211$ & -5.9 & - & 6.0 & $1.58-2.11$ \\
\hline March 31, 1994 & - & - & $59-66$ & 231 & -5.7 & - & 6.0 & $1.57-1.74$ \\
\hline April 2,1994 & - & - & 160 & 272 & -5.4 & - & 6.0 & $1.74-1.82$ \\
\hline April 3, 1994 & - & - & 216 & 295 & -5.2 & - & 6.0 & $1.58-1.68$ \\
\hline April 5, 1994 & - & $147-150$ & $309-319$ & $337-340$ & -4.9 & - & 1.0 & $1.55-2.05$ \\
\hline
\end{tabular}

Other satellites and stars were observed on most nights: this table only refers to the spectra shown in this paper. The air mass difference between the spectra used in most spectral ratios shown in this paper was generally smaller than the full air mass range given here. 
corrections can be judged by the almost perfect removal of the deep $7600 \AA \mathrm{O}_{2}$ - $\mathrm{A}$ band from the satellite spectra in Figures 1 and 3.

After extinction coefficients were determined, star spectra were corrected to the air mass of the satellite spectra, registered in wavelength, and then divided into the satellite spectra to remove solar illumination effects. The star 35 Leo was used as the standard star on March 19, 1993, and 16 Cyg B was used on March 20, 1993. On those nights when standard star spectra were not taken, we ratioed the satellites to each other to look at discrete spectral features. Ratioing to the standard stars removed Fraunhofer lines and telluric features from the satellite spectra very successfully (Figures 1 and 3). Comparison of the Io spectra with the star spectrum in Figure 1 shows that some residual Fraunhofer lines remain, though 16 Cyg B, used for the March 20, 1993 trailing side spectrum, is clearly a better solar analog than 35 Leo, used for the March 19, 1993 leading side spectrum. The $\mathrm{S} / \mathrm{N}$ of the final averaged spectra is between several hundred and 2000 except at short wavelengths (the OSU spectrograph and its CCD are not UV-optimized).

\section{Results}

Io

Figure 1 shows leading and trailing hemisphere spectra of Io. Spectrophotometry at similar orbital longitudes from McFadden et al. [1980] is shown for comparison. No obvious spectral changes since the 1977-1978 McFadden spectra are visible: there are discrepancies in the UV but these may not be real, as larger UV discrepancies are seen for Ganymede
(Figure 3). Note that the McFadden et al. average leading hemisphere spectrum as published in the works by McFadden et al. [1980], Sill and Clark [1982], and Nash et al. [1986] is displaced toward the blue by one data point, and this error has been corrected in Figure 1. The "shelf" in the reflectance spectrum between $5200 \AA$ and $5700 \AA$, first noted by Johnson and McCord [1971], is strikingly apparent, and separates two distinct absorption edges, from 3800 to $5200 \AA$ and from 5700 to $6800 \AA$.

The new Io spectra confirm and highlight discrepancies between Io's reflectance spectrum and published spectra of Io surface models (Figure 2). Though $\mathrm{SO}_{2}$ is known to be abundant on Io's surface, it is spectrally neutral longward of $4000 \AA$ and cannot account for the strong absorptions in the visible spectrum between $4000 \AA$ and $7000 \AA$. Beginning with it Nelson and Hapke [1978], various combinations of sulfur allotropes, compounds, and melt freezes have been proposed to account for the $3700-5200 \AA$ absorption edge. Two examples are shown here: the Moses and Nash [1991] model containing $\mathrm{SO}_{2}$, sulfur melt freezes, and quenched red sulfur glass, and the novel model of Hapke [1989], which includes polysulfur oxide, $\mathrm{SO}_{2}, \mathrm{~S}_{2} \mathrm{O}$, and basalt powder. Neither model matches the $5200-\AA$ to $5700-\AA$ shelf and $5700-\AA$ to $6900-\AA$ edge particularly well, though the Hapke model is somewhat better because $\mathrm{S}_{2} \mathrm{O}$ has a 5700 - to $6800-\AA$ absorption edge similar to that on Io. Nash [1993] suggests that the $5200-\AA$ to $6800-\AA$ spectrum shape is due to the presence of both $\mathrm{Na}_{2} \mathrm{~S}$ and $\mathrm{S}_{2} \mathrm{O}$ on Io, and by including both these components he is able produce a better match to Io's spectrum in this region than either of the matches shown in Figure 2.

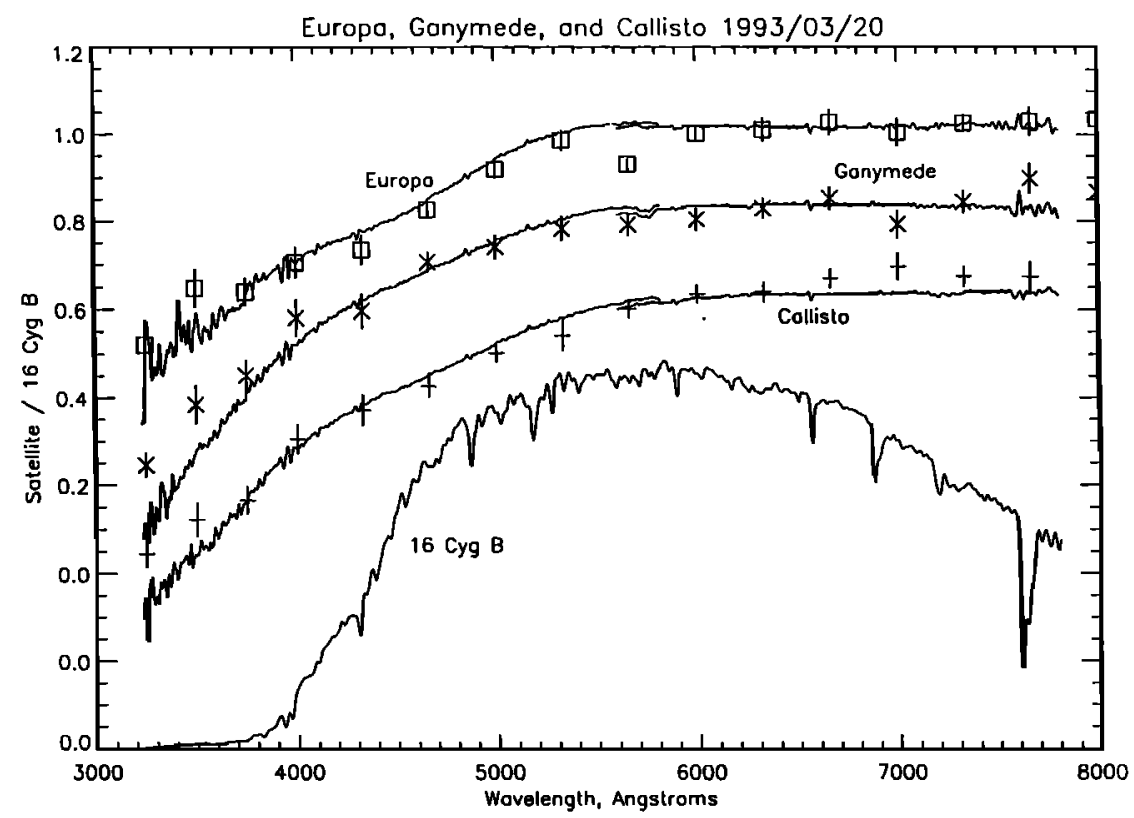

Figure 1. Leading and trailing hemisphere spectra of Io, ratioed to solar-type standard stars and converted to geometric albedo (including the opposition effect) by scaling by the Io $\mathrm{V}$ magnitude at the appropriate longitude [from Morrison and Morrison, 1977]. Also shown is the unratioed spectrum of the solar-type standard, 35 Leo, which shows the low intrinsic signal level in the ultraviolet. Fraunhofer and telluric lines, seen in the star spectrum, are removed quite successfully from the ratio spectra, with the exception of the $5170 \AA \mathrm{Mg}$ line in the March 19, 1993 data, which is apparently stronger in 35 Leo (used as the solar analog on that date) than in the sun. The star 16 Cyg B, used as the solar analog on March 20, 1993, is a much closer match here. Na-D emission at $5893 \AA$ is seen in the trailing side spectrum. For comparison, 1977-1978 spectrophotometry of Io by McFadden et al. [1980] is also shown. 


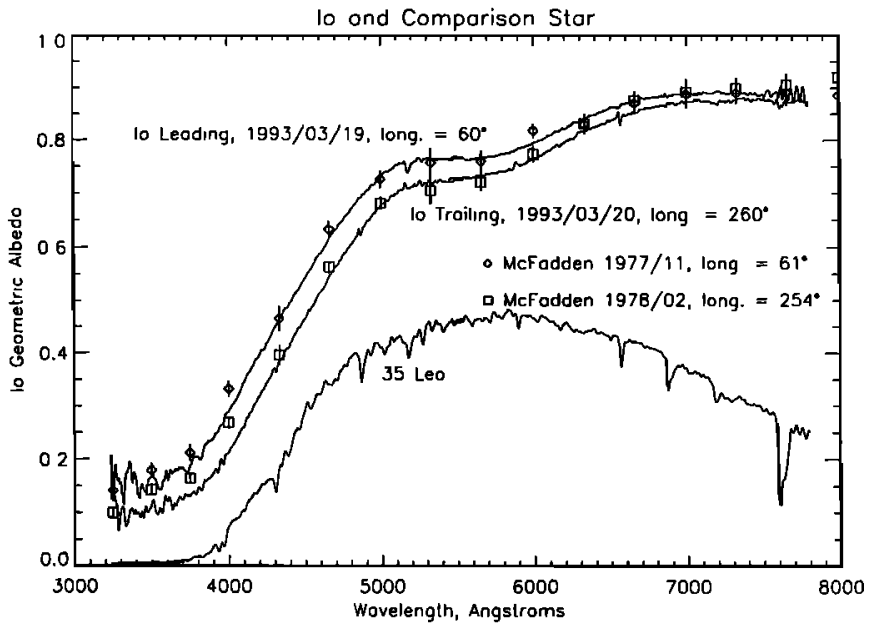

Figure 2. A composite spectrum of Io's leading hemisphere, including our March 19, 1993 CCD spectra at longer wavelengths, and short-wavelength data from Nelson and Hapke [1978], which has lower noise than the CCD spectra below $4000 \AA$. The Io spectrum is compared to two contrasting published surface composition models. The Hapke [1989] model consists of $50 \%$ polysulfur oxide, $10 \% \mathrm{SO}_{2}$, $15 \% \mathrm{~S}_{2} \mathrm{O}$, and $25 \%$ basalt powder. The Moses and Nash [1991] model consists of $15 \% \mathrm{SO}_{2}, 50 \% 453 \mathrm{~K}$ sulfur melt freeze, $20 \%$ quenched red sulfur glass, and $15 \% 393 \mathrm{~K}$ sulfur melt freeze. Model curves are digitized from the literature, and slight variations from the original may exist. The feature in the Io spectrum at $5170 \AA$ is an incompletely cancelled solar Fraunhofer line.

The lack of obvious spectral changes since 1978, along with Io's long-term photometric stability [Morrison et al., 1979] and the stability of its large-scale albedo patterns [Sartoretti et al., 1995; Spencer et al., 1994], suggests that volcanic resurfacing tends to overpaint areas with more material of the same composition, though the Voyager images clearly show local, short-term exceptions to this rule.

\section{Icy Satellites}

Figure 3 shows the March 20, 1993 reflectance spectra for Europa, Ganymede, and Callisto. Also shown is spectrophotometry by McFadden et al. [1980] at very similar orbital longitudes. There are significant discrepancies between the two data sets in the UV, particularly on Ganymede, which are not yet understood but may be due to the limitations of our extinction correction procedures. The shape of the continuum on Ganymede and Callisto is remarkably similar despite the difference in albedo, but Europa is very different, with a much flatter continuum longward of $5400 \AA$ and a fairly sudden turndown at shorter wavelengths. This shows that the nonice darkening agent on Europa is different in composition from that on Ganymede and Callisto. A simple model in which a single exogenic source of nonice darkening material accumulates on the satellite surfaces over time, as might be suggested by the inverse age/albedo correlation on the icy satellites, may work for Ganymede and Callisto, but Europa's darkening agent must be different because of its different spectrum.

\section{Europa's Continuum: Sulfur?}

Given the known presence of $\mathrm{S}$ and $\mathrm{SO}_{2}$ on Europa from UV spectroscopy [e.g., Lane et al., 1981; Sack et al., 1992], it seems reasonable to suggest that magnetospherically implanted $\mathrm{S}$ ions or compounds created therefrom might also influence the shape of the spectrum at longer wavelengths. Figure 4a compares the reflectance of Europa with the reflectance of several sulfur-bearing materials, including pure sulfur, $\mathrm{SO}_{2}$, and $\mathrm{NaHS}$ after low energy proton irradiation. Simple linear mixes of these materials, plus a neutral continuum, were made to attempt to match the Europa spectrum. These mixes are shown in Figure 4b. From these simple models we can infer that the $\mathbf{5 0 0 0}-\AA$ shoulder is extremely well matched by orthorhombic $\alpha$-sulfur, and that the slope change below $3800 \AA$, if real (spectrum quality is poorer in the UV), may be related to the presence of $\mathrm{SO}_{2}$ frost. We also note that the $\mathrm{SO}_{2}$ shoulders at 3400 and $3900 \AA$ are still prominent in the mixtures, but not observed on Europa. Spectra of medium grained $\mathrm{SO}_{2}$ frosts as shown by $\mathrm{Nash}$ et al. [1980] exhibit less of a well-defined absorption band between 3500 and $4000 \AA$. Inclusion of more coarse-grained $\mathrm{SO}_{2}$ would retain the absorption edge at $3800 \AA$, but the pronounced peak at $3400 \AA$ would disappear, providing a better match to the Europa spectrum than the models presented here. Inclusion of a small amount of $\mathrm{NaHS}$, which has the short wavelength edge of the conduction band shifted from that of the sulfur tends to flatten the $3900 \AA$ shoulder of $\mathrm{SO}_{2}$ and provides a slightly better match at that wavelength. A spectrum of $\mathrm{Na}_{2} \mathrm{O}_{2}$ recently shown by $\mathrm{Nash}$ [1993] has similar characteristics to that of the $\mathrm{NaHS}$, but with a better defined shoulder beyond $5000 \AA$. Inclusion of this material might further improve the match to the Europa spectrum.

The $\alpha$-sulfur spectrum in Figure 4 was taken at room temperature, and solid sulfur has a known reduction in the

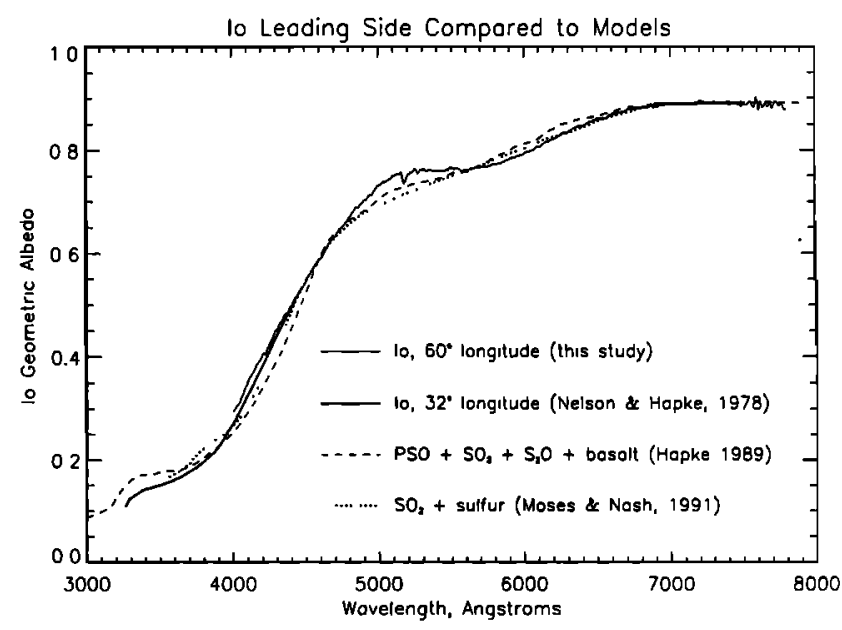

Figure 3. Europa, Ganymede, and Callisto, ratioed to 16 Cyg B, on March 20, 1993. A raw star spectrum is shown for comparison. For best comparison of continuum shapes, all satellite spectra are scaled to 1.0 at $5630 \AA$ and then offset vertically in increments of 0.2 . Note the overlap between the data at the two grating positions near 5700 $\AA$. Also shown is spectrophotometry by McFadden et al. [1980] at very similar orbital longitudes. Orbital longitudes for each satellite are as follows. Europa $81^{\circ}$ (CCD), $80^{\circ}$ (McFadden); Ganymede $252^{\circ}$ and $251^{\circ}$; Callisto $70^{\circ}$ and $62^{\circ}$. Note the similarity of Ganymede and Callisto, the very different shape of Europa's continuum, and the weak $5773-\AA$ and $6275-\AA$ features on Ganymede. 

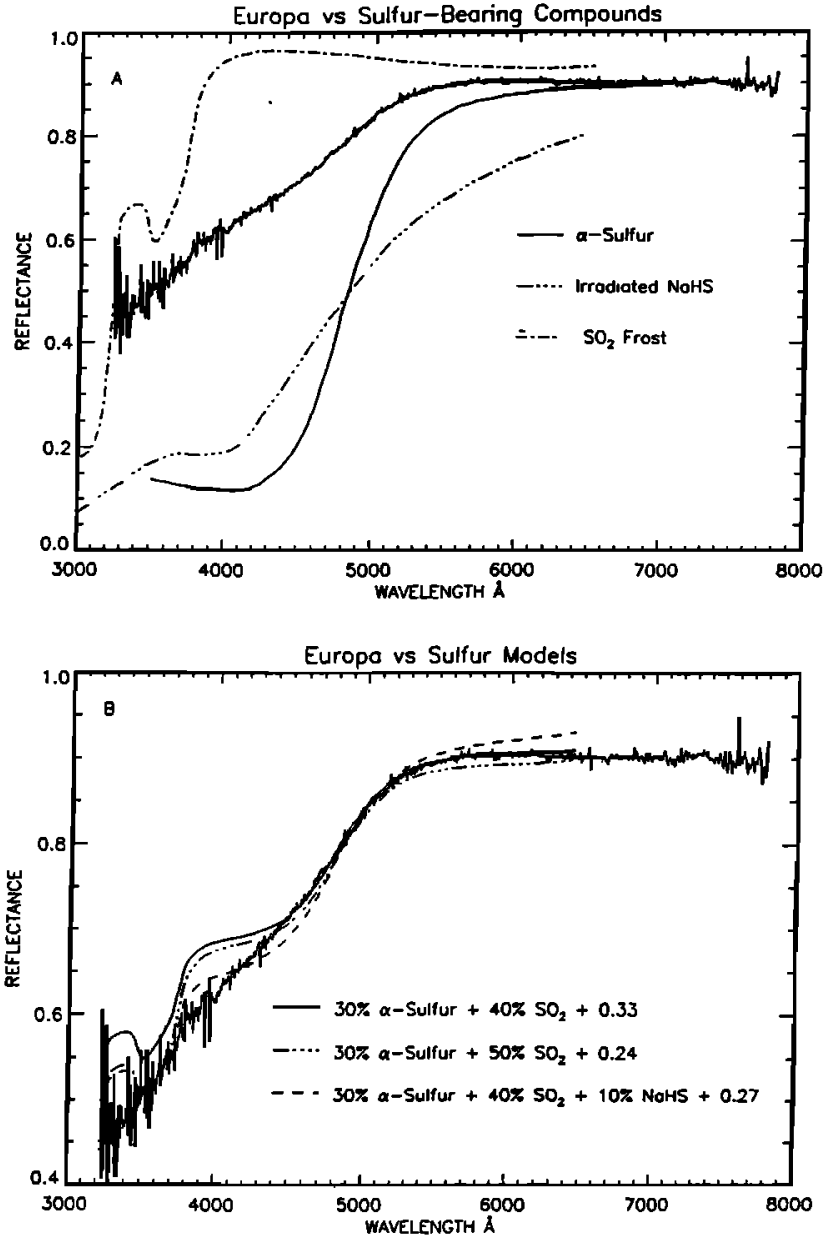

Figure 4. (a) March 20, 1993 spectrum of Europa's leading side compared with $\alpha$-sulfur from Moses and Nash [1991], irradiated NaHS from Nash and Nelson [1979], and $\mathrm{SO}_{2}$ frost from Soderblom et al. [1980]. Curves were digitized from the literature, and slight variations from the original may exist. The $\mathrm{SO}_{2}$ curve is offset 0.15 upward for clarity. (b) Comparison of the Europa spectrum to three linear combinations of the materials in Figure $4 a$, plus a constant value representing a neutral continuum. The turnover at $5000 \AA$ is matched perfectly, though all models show more structure at shorter wavelengths than is seen on Europa.

wavelength of the conduction band edge with decreasing temperature [e.g., Moses and Nash, 1991, and references therein]. We might therefore expect the Europa $5000 \AA$ turnover to be at shorter wavelengths than the lab spectrum, as is seen on Io (Figure 1). However, in contrast to Io, the sulfur on Europa is probably not present as a continuous solid but as a contaminant in the abundant water ice. The presence of water ice may result in donor sites for electrons which lower the energy required for electrons to move into the conduction band, which effectively shifts the spectral conduction band edge to longer wavelengths, counteracting the temperature shift. As an example, a number of sulfides exhibit conduction band edges at longer wavelengths than observed in pure sulfur [Hunt et al., 1971]. Alternatively, polymers of $\mathrm{S}_{2} \mathrm{O}$ with $\mathrm{SO}_{2}$, as suggested by Sill and Clark [1982] to account for the colors on Io, may be present and contributing to the visual color of Europa.
In any case it seems quite likely that some type of sulfurbearing compound is responsible for the $5000-\AA$ turnover seen in the spectrum of Europa, and possible that $\mathrm{SO}_{2}$ frosts may yield the possible change in slope below 3800 $\AA$. It is possible that the sulfur is endogenic, as the 5000 $\AA$ turnover is seen on both the leading and trailing hemispheres of Europa [Nelson and Hapke, 1978], and McEwen [1986] showed that the obvious exogenic absorption pattern on Europa predominantly affects the ultraviolet reflectance with a strong concentration on the trailing hemisphere. The endogenic lineaments are predominantly darker and redder than the rest of the surface in the Voyager images [Buratti and Golombek, 1988], and thus probably have a stronger $5000 \AA$ turnover than the rest of the surface. The combined effects of reduced (or different) resurfacing and reduced magnetospheric impact inferred for Ganymede and Callisto can then explain the continuum shape difference between the three satellites in the visible part of the spectrum.

\section{Discrete Features on Ganymede}

Figure 3 shows that the leading-side Europa and Callisto spectra are smooth to the limit of resolution. Data from other nights show that the trailing hemisphere spectra of both these objects are equally smooth. However, on Ganymede, a previously unknown shallow, narrow, absorption feature is apparent at $5773 \AA$, with a probable shallower feature at $6275 \AA$. The narrowness, sharpness, and visible wavelength of the Ganymede features distinguish them from any spectral reflectance feature previously seen on solid surfaces in the solar system, though some asteroids appear to show rather less well-defined discrete shallow features in their visible spectra [Luu and Jewitt 1990; Vilas and Gaffey 1989; Vilas et al., 1993]. However, we are convinced of the reality of the 5773- $\AA$ feature for the following reasons: (1) it appears at the same wavelength independent of the grating used or the grating tilt, so it is not dependent on position on the CCD or on the specific path through the spectrograph; (2) it is seen consistently on Ganymede, and never on the other satellites; (3) spectra of Jupiter show no feature here, and none of the Jupiter $\mathrm{CH}_{4}$ features at longer wavelengths appear in the satellite spectra, so it is not due to contamination of the spectrum by stray light from Jupiter; (4) it shows no correlation with Fraunhofer lines, and appears in ratios of Ganymede to different standard stars and in ratios to other satellites, so it is not a stellar feature; (5) following our discovery of the feature, it was noted, with lower $S / N$ but consistent appearance, in Ganymede/Callisto ratio spectra taken by W. Grundy et al. (personal communication, 1993) at the Catalina 61-inch telescope in March 1992.

The $6275 \AA$ feature is shallower and has more variation in its appearance (possibly because it overlaps a weak telluric feature due to $\mathrm{H}_{2} \mathrm{O}$ near $6300 \AA$ ), but is persistent enough that we were almost certain that it was also real even before we were able to identify it (see below).

Figure 5 shows most of our Ganymede spectra in the 5000- to $7000-\AA$ region, ratioed to another satellite for the best cancellation of Fraunhofer lines. The 5773- $\AA$ feature is present in all trailing-side spectra, and probably in the April 22, 1993 spectrum of the leading side, but not in the 1994 leading-side spectra. Whether the discrepancy on the leading side between 1993 and 1994 is due to time variability, sudden changes in band depth with orbital longitude, or 


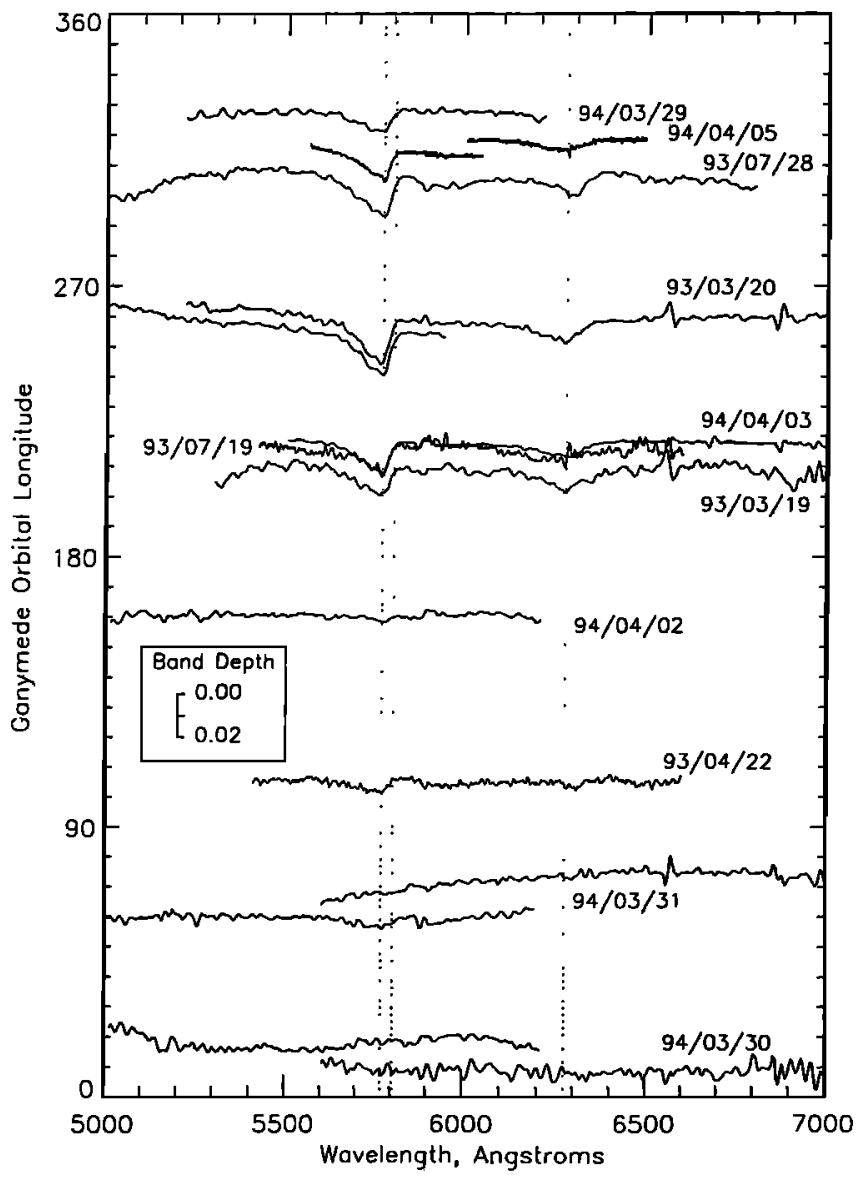

Figure 5. Most of our Ganymede spectra in the 5000- to $7000-\AA$ region, ratioed to Callisto (or Europa in the case of July 28, 1993). The spectra have been ratioed to a linear least squares fit to remove overall slopes, except that a cubic fit was used for the July 28, 1993 spectrum and a quadratic fit for the April 2, 1994 spectrum. Spectra are shown to the same scale, given in the inset box, and are offset vertically so that the vertical position at $5810 \AA$ gives the central longitude of Ganymede for each spectrum. The vertical dashed lines indicate the wavelengths 5773,5805 , and $6275 \AA$.

simply limitations of the data is not yet clear. The $6275-\AA$ feature, though more variable, is present in most trailingside spectra but not on the leading side. Figure 6 further illustrates the variation in the depth of the 5773- $\AA$ band with orbital longitude: band depth is sharply peaked in the center of the trailing hemisphere and falls off steeply away from there.

On April 5, 1994, we obtained spectra of the satellites with a higher resolution grating (dispersion $1 \AA$ pixel $^{-1}$, resolution $3 \AA$ ), and these are shown in Figure 7. This figure provides the best view of the $5773-\AA$ feature yet available. The $5773-\AA$ feature has minimum reflectance at $5773 \pm 1$ $\AA$. It is asymmetric, with a gradual return to the continuum over about $180 \AA$ on the short-wavelength side, and a sharp long-wavelength edge returning to the continuum in $32 \AA$, at $5805 \AA$. No convincing fine structure is seen at $3 \AA$ resolution, though the minimum and long-wavelength edge are sharp to the limit of resolution.

We also looked at the $6275-\AA$ Ganymede feature at $3-\AA$ resolution on April 5, 1994 (Figure 5). Unlike the $5773 \AA$ feature, no sharp structure was seen, at least at the orbital longitude observed on that night $\left(318^{\circ}\right)$.

\section{Origin of the Ganymede Features: $\mathrm{O}_{2}$}

The 5773- and $6275-\AA$ Ganymede features are matched almost perfectly in wavelength and shape by the two strongest visible-wavelength features in the spectrum of solid diatomic oxygen [Landau et al., 1962], (Figures 7 and 8). The closeness of the match, our extensive and unsuccessful searches for other matches, and the fact that nextstrongest solid oxygen absorption band in our wavelength range, at $5320 \AA$, is weaker than the $6275 \AA$ feature by an order of magnitude in all of the crystallographic phases of $\mathrm{O}_{2}$ [Landau et al., 1962], provides convincing evidence that the identification is correct. The solid oxygen features are produced by simultaneous $a^{1} \Delta_{g} \rightarrow X^{3} \Sigma_{g}^{-}$transitions in two adjacent $\mathrm{O}_{2}$ molecules: the $5770-\AA$ feature is the 1-0 band and the $6275-\AA$ feature is the $0-0$ band. Both features are also seen in liquid $\mathrm{O}_{2}$, and in $\mathrm{O}_{2}$ gas when pressures are high enough to produce a sufficient number of $\mathrm{O}_{2}$ molecules during collisions [Pearse and Gaydon, 1976].

The presence of these bands on Ganymede is suprising, as at its freezing point, $54 \mathrm{~K}$, the vapor pressure of solid $\mathrm{O}_{2}$ is 1.3 mbars [Weast, 1974], $10^{8}$ times greater than the occultation upper limit to Ganymede's surface pressure [Broadfoot et al., 1979]. The coldest daytime temperature measured on Ganymede by the Voyager infrared interferometer spectrometer (IRIS) instrument was $71 \mathrm{~K}$, within $8^{\circ}$ of the South Pole [Spencer, 1987] Generation of the bands in gaseous $\mathrm{O}_{2}$ is out of the question, as the bands are much stronger on Ganymede than in the Earth's atmosphere. We are left with the possibility that the $\mathrm{O}_{2}$ molecules generating the features are somehow trapped in the surface materials, perhaps as a clathrate in the surface water ice, though the trapped $\mathrm{O}_{2}$ molecules must be close enough together to allow simultaneous electronic transitions in two adjacent molecules. The spectral match to Ganymede is particularly good for $\beta$

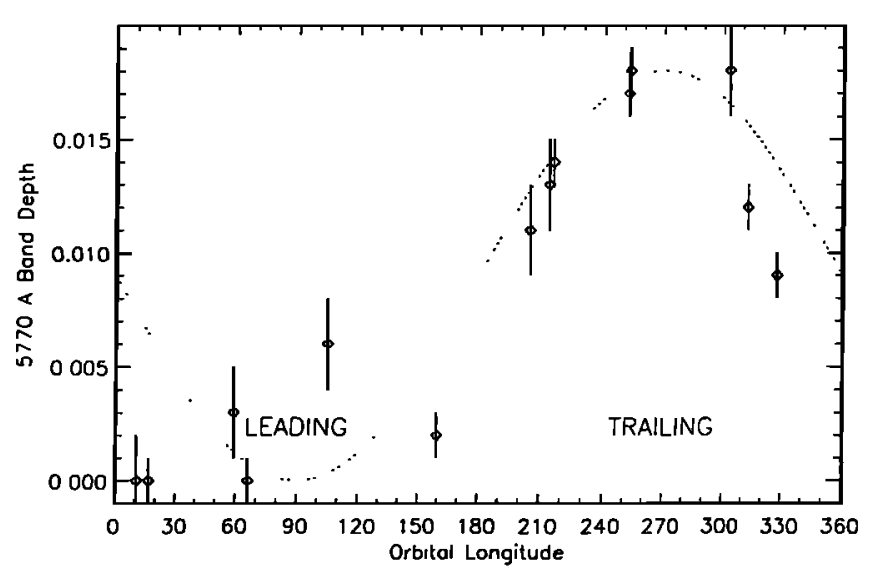

Figure 6. Depth of Ganymede's 5773- $\AA$ feature as a function of orbital longitude, from the spectra in Figure 5. Depth is defined as the difference in reflectance between 5773 and $5810 \AA$, divided by the $5810-\AA$ reflectance. A sinusoid, with a peak in the center of the trailing hemisphere, is shown for reference (dotted line): the band depth is quite symmetrical about the center of the trailing hemisphere but drops off more rapidly than a sinusoid away from the peak longitude. 


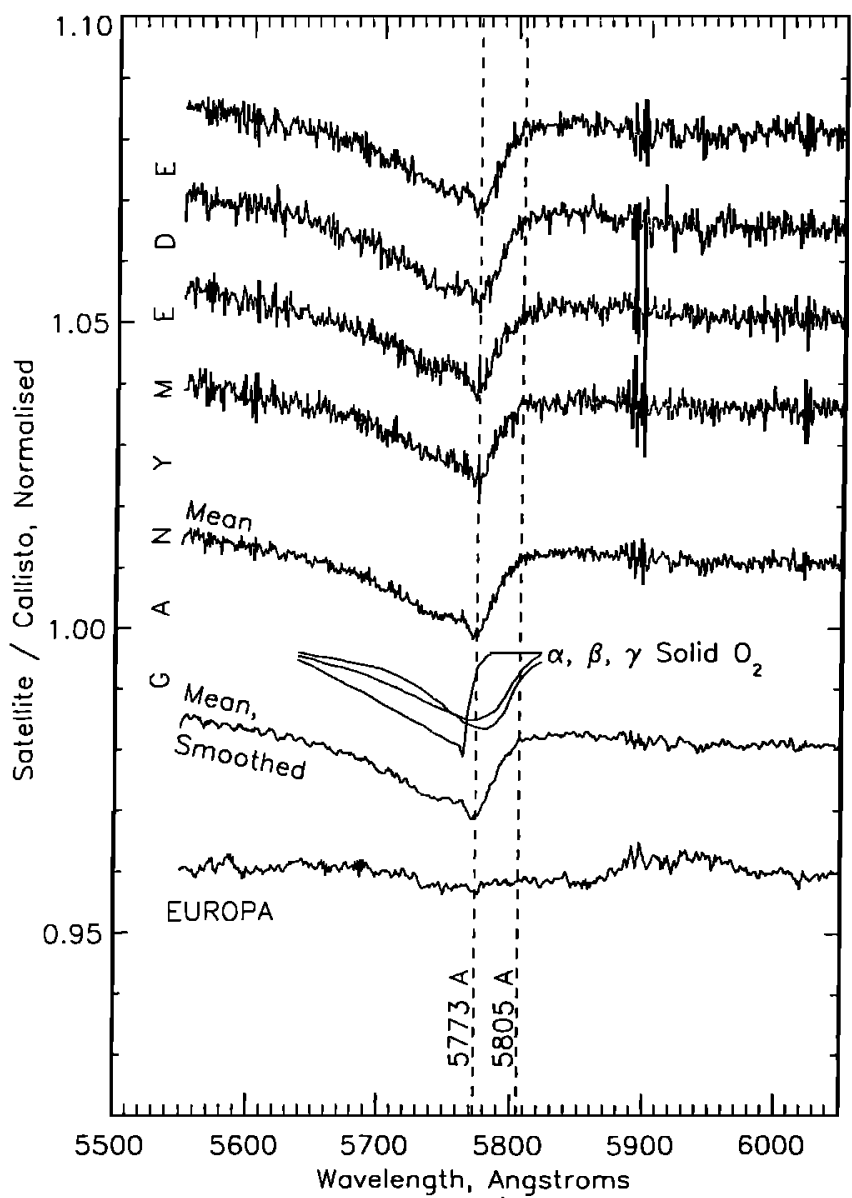

Figure 7. High spectral resolution April 5, 1994 Ganymede and Europa spectra, ratioed to Callisto. Ratio spectra are divided by a linear fit and then offset vertically. At the top are four different Ganymede spectra, each (with one exception) divided by a different Callisto spectrum, and below these are the mean of the four spectra and the mean after a three-point boxcar smooth has been applied. The lowest spectrum is the smoothed sum of two independent Europa/Callisto ratio spectra. This is of lower quality due to Europa's closeness to Jupiter on this date, but shows the absence of the 5773- $\AA$ feature on either Europa or Callisto. Artifacts near $5890 \AA$ are due to imperfect cancellation of the Na-D Fraunhofer lines. Also shown are the spectra of the $\alpha, \beta$, and $\gamma$ forms of solid $\mathrm{O}_{2}$ ( $\alpha$ is the lowermost and $\gamma$ the uppermost curve to the left of the absorption maximum), from Landau et al. [1962]: their absorption intensity is subtracted from a constant to mimic the Ganymede reflectance spectra.

$\mathrm{O}_{2}$ (Figures 7 and 8 ), which may provide a clue as to the environment of the $\mathrm{O}_{2}$ molecules on Ganymede's surface. Landau et al. give a band-integrated absorption coefficient of $1.1 \times 10^{4} \mathrm{~cm}^{-2}$ for the $5773-\AA$ band in $\alpha-\mathrm{O}_{2}$, so the Ganymede $5773-\AA$ feature's maximum equivalent width of about $3 \mathrm{~cm}^{-1}$ requires the equivalent of about a $3 \mu \mathrm{m}$ pathlength in solid $\mathrm{O}_{2}$ on the trailing hemisphere. The bands of $\beta$ and $\gamma \mathrm{O}_{2}$ are weaker and would require up to 10 times as much surface $\mathrm{O}_{2}$ to reproduce the Ganymede features. However, as none of these forms of $\mathrm{O}_{2}$ can actually be stable on Ganymede, it is not yet possible to give a real quantitative abundance estimate for Ganymede's molecular oxygen.

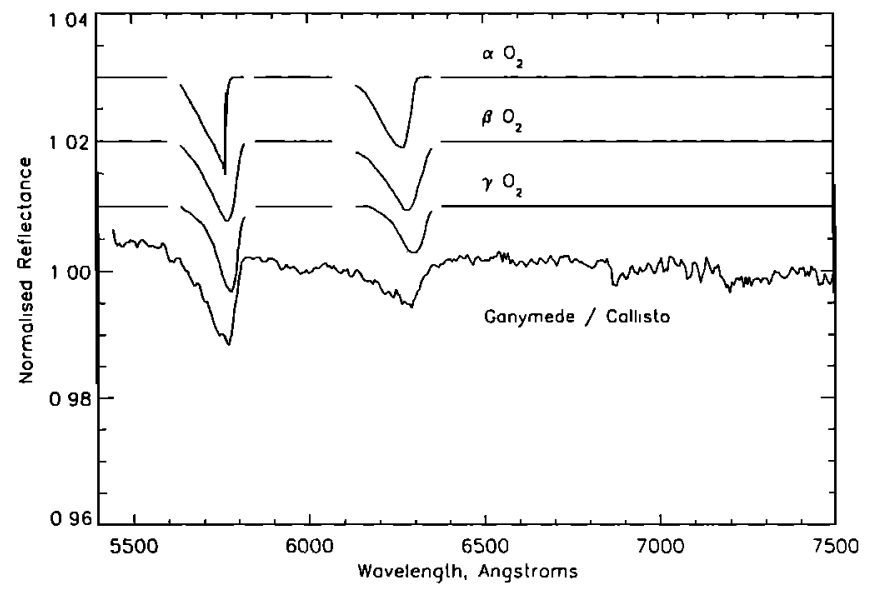

Figure 8. April 3, 1994 Ganymede/Callisto ratio spectrum, with continuum slope removed by dividing by a quadratic fit to the ratio, compared to the spectra of the $\alpha, \beta$, and $\gamma$ forms of solid $\mathrm{O}_{2}$, from Landau et al. [1962]: their plotted absorption intensities in the two bands are subtracted from a constant to mimic the Ganymede reflectance spectra, and horizontal lines at the constant value are plotted between the bands to emphasize the lack of other features in the $\mathrm{O}_{2}$ spectrum in this wavelength range. Relative strength of the two bands is correct for each phase. Features in the Ganymede/Callisto ratio near 6880 and $7200 \AA$ are due to imperfectly cancelled Fraunhofer lines.

Note that Nelson et al.. [1987] presented evidence for molecular oxygen in the form of ozone on Ganymede's trailing side. They noted that Ganymede's trailing hemisphere has an additional source of absorption at wavelengths below $2800 \AA$ that is absent or reduced on the leading side, and suggested $\mathrm{O}_{3}$ resulting from oxygen implantation in an ice matrix as a possiblity. Liquid $\mathrm{O}_{2}$ shows continuous absorption below $2609 \AA$ [Pearse and Gaydon, 1976] so it is possible that the condensed $\mathrm{O}_{2}$ seen at visible wavelengths also contributes to the UV absorption seen in the IUE spectra.

The concentration of the oxygen on Ganymede's trailing side suggests that it is generated by magnetospheric bombardment, either by direct implantation of oxygen ions or by processing of surface $\mathrm{H}_{2} \mathrm{O}$, but the absence on the trailing sides of Europa and Callisto remains a mystery. Perhaps the high magnetospheric fluxes on Europa favor destruction of $\mathrm{O}_{2}$ over its formation, or endogenic resurfacing removes the oxygen, and ion fluxes may be too low for significant formation on Callisto, but this is speculation. Laboratory work may shed light on these questions in the future. It will also be interesting to investigate what, if any, relationship exists between the condensed $\mathrm{O}_{2}$ on Ganymede and the gaseous $\mathrm{O}_{2}$ recently detected at Europa [Hall et al., 1995].

Acknowledgments. The observations of Io were first suggested by Doug Nash, and Mark Wagner obtained the July 19, 1993 spectra. Jane Spencer, Christophe Dumas, Laura Woodney, and Amor Angara provided assistance at the telescope, and Ray Bertram and Jeff Pier gave up twilight time for the unscheduled 1993 follow-up observations. Jeff Bell and Lucy McFadden provided help with the 1978 McFadden et al. observations. Mark Wagner, Dave Schleicher, Marc Buie, Bill McKinnon, and many others offered useful comments and advice. The observations and analysis were supported by NASA grant NAGW-2785 and the Galileo NIMS project. 


\section{References}

Broadfoot, A. L., et al. Extreme ultraviolet observations from Voyager 1 encounter with Jupiter, Science, 204, 979-982, 1979.

Buratti, B. J., and M. P. Golombek, Europa: Geological implications of spectrophotometry, Icarus, 75, 437-449, 1988.

Hall, D. T., D. F. Strobel, P. D. Feldman, M. A. McGrath, and $H$. A. Weaver, Detection of an oxygen atmosphere on Jupiter's moon Europa, Nature, 373, 677-679, 1995.

Hapke, B., The surface of Io: A new model, Icarus, 79, 56-74, 1989.

Hardorp, J., The Sun among the stars, III, Energy distributions of 16 northern G-type stars and the solar flux calibration, Astron. Astrophys., 91, 221-232, 1980.

Hunt, G. R., J. W. Salisbury, and C. J. Lenhoff, Visible and near-infrared spectra of mineral and rocks, IV, Sulphides and sulphates, Mod. Geol., 3, 1-14, 1971.

Johnson, T. V., Galilean satellites: Narrowband photometry 0.30 to 1.10 microns, Icarus, 14, 94-111, 1971.

Johnson, T.V. and R. B. McCord, Galilean satellites: The spectral reflectivity 0.30-1.10 microns, Icarus 19, 37-42, 1970.

Landau, A., E. J. Allin, and H. L. Welsh, The absorption spectrum of solid oxygen in the wavelength region from $12,000 \AA$ to $3300 \AA$, Spectrochim. Acta, 18, 1-19, 1962.

Lane, A. L., R. M. Nelson, and D. L. Matson, Evidence for sulfur implantation in Europa's UV absorption band, Nature, 292, 38-39, 1981.

Luu, J. X., and D. C. Jewitt, CCD spectra of asteroids, I, Nearearth and 3:1 resonance asteroids, Astron. J., 99, 1985-2011, 1990.

McEwen, A. S., Exogenic and endogenic albedo and color patterns on Europa, J. Geophys. Res., 91, 8077-8097, 1986.

McFadden, L. A., J. F. Bell, and T. B. McCord, Visible spectral reflectance measurements $(0.33-1.1 \mu \mathrm{m})$ of the Galilean satellites at many orbital phase angles, Icarus, 44, 410-430, 1980.

Morrison, D., and N. Morrison, Photometry of the Galilean satellites, in Planetary Satellites, edited by J. Burns, pp. 363-378, Univ. of Ariz. Press, Tucson, 1977.

Morrison, D., D. Pieri, J. Veverka, and T.V. Johnson, Photometric evidence on long-term stability of albedo and color markings on Io, Nature, 280, 753-755, 1979.

Moses, J. I., and D. B. Nash, Phase transformations and spectral reflectance, of solid sulfur: Can metastable sulfur allotropes exist on Io?, Icarus, 89, 277-304, 1991.

Nash, D. B., The case for $\mathrm{Na}_{2} \mathrm{~S}$ on Io's surface: sulfide volcanism?, paper presented at Io: An International Conference, San Juan Institute, San Juan Capistrano, June 1993.

Nash, D. B., and R. M. Nelson, Spectral evidence for sublimates and adsorbates on Io, Nature, 280, 763-766, 1979.
Nash, D. B., F. P. Fanale, and R. M. Nelson, $\mathrm{SO}_{2}$ frost: UVvisible reflectivity and limits on Io's surface coverage, Geophys. Res. Lett., 7, 665-668, 1980.

Nash, D. B., M. H. Carr, J. Gradie, D. M. Hunten, and C. F. Yoder, Io, in Satellites, edited by J. Burns and M. Matthews, pp. 629-688, Univ. of Ariz. Press, Tucson, 1986.

Nelson, R. M., and B. W. Hapke, Spectral reflectivities on the Galilean satellites and Titan, 0.32 to 0.86 micrometers, Icarus, 36, 304-329, 1978.

Nelson, R. M., A. L. Lane, D. L. Matson, G. J. Veeder, B. J. Buratti, and E. F. Tedesco, Spectral geometric albedos of the Galilean satellites from 0.24 to 0.34 micrometers: Observations with the International Ultraviolet Explorer, Icarus, 72, 358-380, 1987.

Pearse, R. W. B., and H. G. Gaydon, Identification of Molecular Spectra, Chapman and Hall, New York, 1976.

Sack, N. J., R. E. Johnson, J. W. Boring, and R. A. Baragiola, The effect of magnetospheric ion bombardment on the reflectance of Europa's surface, Icarus, 100, 534-540, 1992.

Sartoretti, P., M.A McGrath, A.S. McEwen, and J.R. Spencer, Post-Voyager brightness variations on Io J. Geophys. Res., 100, 7523-7530, 1995.

Sill, G. T., and R. N. Clark, Composition of the surfaces of the Galilean satellites, in Satellites of Jupiter, edited by D. Morrison, pp. 174-212, Univ. of Arizona, Tucson, 1982.

Soderblom, L., et al., Spectrophotometry of Io: Preliminary Voyager 1 results, Geophys. Res. Lett., 7, 963-966, 1980.

Spencer, J. R., The surfaces of Europa, Ganymede, and Callisto: An investigation using Voyager IRIS infrared spectra, Ph.D. dissertation, U. Arizona, 1987.

Spencer, J. R., M. A. McGrath, P. Sartoretti, and A. S. McEwen Post-repair HST imaging of Io: Surface changes and spectral anomalies, Bull. Am. Astron. Soc., 26, 1994.

Vilas, F., and M. Gaffey, Phyllosilicate absorption features in main-belt and outer-belt asteroid reflectance spectra, Science, 246, 790-792, 1989.

Vilas, F., E.C. Hatch, S.M. Larson, S.R. Sawyer, and M.J. Gaffey, Ferric iron in primitive meteorites: $\mathrm{A} 0.43 \mu \mathrm{m}$ absorption feature, Icarus, 102, 225-231, 1993.

Weast, R. C. (Ed.), Handbook of Chemistry and Physics, CRC Press, Cleveland, 1974.

W. M. Calvin, U.S. Geological Survey, 2255 N. Gemini Drive, Flagstaff, AZ 86001. (e-mail: wcalvin@hulk.wr.usgs.gov)

M. J. Person and J. R. Spencer, Lowell Observatory, 1400 W. Mars Hill Road, Flagstaff, AZ 86001. (e-mail: spencer@lowell.edu)

(Received May 24, 1994; revised April 13, 1995; accepted May 10, 1995.) 